\title{
Reconstruction Method for Nonconcyclic Dual-Source Circular Cone-Beam CT with a Large Field of View
}

\author{
Ming Chen, ${ }^{1}$ Gang Li, ${ }^{1}$ Weiwei Qi, ${ }^{2}$ Jing Zou, ${ }^{3}$ and Yong-guo Zheng ${ }^{1}$ \\ ${ }^{1}$ College of Information Science and Engineering, Shandong University of Science and Technology, Qingdao 266590, China \\ ${ }^{2}$ The First Research Institute of the Ministry of Public Security, Beijing 100048, China \\ ${ }^{3}$ State Key Laboratory of Precision Measuring Technology and Instruments, Tianjin University, Tianjin 300072, China
}

Correspondence should be addressed to Gang Li; ligangccm@163.com

Received 19 November 2012; Revised 11 February 2013; Accepted 13 February 2013

Academic Editor: Yingwei Zhang

Copyright (C) 2013 Ming Chen et al. This is an open access article distributed under the Creative Commons Attribution License, which permits unrestricted use, distribution, and reproduction in any medium, provided the original work is properly cited.

\begin{abstract}
In industrial computed tomography (CT), it is often required to inspect large objects whose size is beyond a reconstructed field of view (FOV). Some multiscan modes have been developed to acquire the complete CT projection data for a larger object using small panel detectors. In this paper, we give a non-concyclic dual-source circular cone-beam scanning geometry based on the idea of multiscan modes and propose a backprojection-filtration-based (BPF) reconstruction algorithm without data rebinning. Since the FOV calculated according to this nonconcyclic dual-source circular CT scanning geometry is larger than cardiac dual-source CT scanning geometry, our method can reconstruct larger horizontal slices (i.e., the slices perpendicular to rotation axis) than cardiac dual-source CT. The quality of CT images is expected to be superior to those obtained using larger panel detectors. The simulation results have indicated that $\mathrm{CT}$ images obtained by the proposed method are satisfying.
\end{abstract}

\section{Introduction}

In industrial $\mathrm{CT}$, it is often required to inspect large objects whose size is beyond a field of view (FOV) calculated according to CT scanning geometry, where the maximum horizontal (i.e., perpendicular to rotation axis) FOV is usually fixed in CT system. When a slice of the inspected object cannot be completely covered within the FOV of the cone beam, complete projection data passing through the object cannot be obtained. However, conventional CT reconstruction algorithm, for example, filtered backprojection (FBP), requires the complete projection data. Therefore, those conventional scan modes and reconstruction methods can not be used. To solve this problem, several multiscan modes have been developed to acquire the complete projection data. Of them, the rotation-translation-translation (RTT) multiscan mode $[1,2]$ and rotation-translation (RT) multiscan mode $[3,4]$ are typical. In fact, RT and RTT multiscan modes not only acquire completed projection data, but can also reduce the differences of the flux intensities detected by different detector cells.
For fan-beam RT multiscan modes, the reconstruction algorithm based on backprojection- filtration (BPF) was proposed by Chen et al. $[5,6]$, which can exactly reconstruct the images and does not introduce data rebinning. BPF algorithm, as the basis of our work, was first proposed by Zou and Pan [7] to exactly reconstruct the images for helical cone beam CT. After that, an explicit BPF formula for 2D image reconstruction was proposed by Noo et al. [8] using the finite inversion formula of Hilbert transform.

In this work, we give a nonconcyclic dual-source circular cone beam scanning geometry based on the idea of RT multiscan scan mode and propose a BPF-based reconstruction formula. Since The FOV calculated according to this nonconcyclic dual-source circular CT scanning geometry is larger than cardiac dual-source CT scanning geometry, our method can reconstruct larger horizontal slices. The quality of CT images reconstructed is expected to be superior to those obtained using larger panel detectors. The simulation results have indicated that $\mathrm{CT}$ images obtained by the proposed algorithm are satisfying. Since we make use of the approximate idea of Feldkamp (FDK) algorithm [9] in 


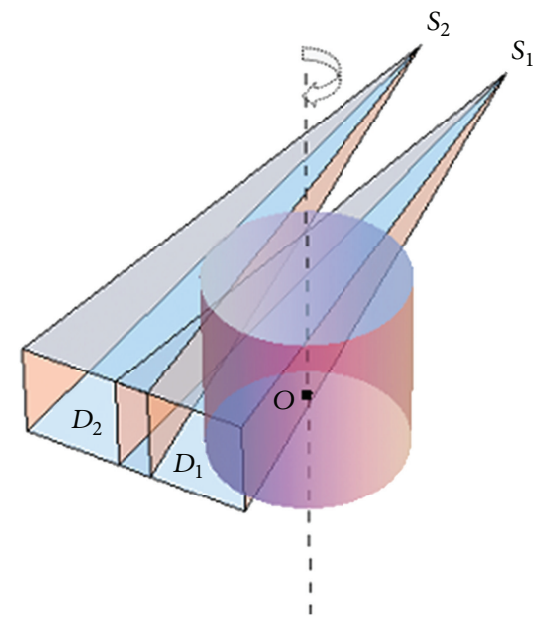

FIgURE 1: The nonconcyclic dual-source circular cone beam scanning geometry with a larger FOV.

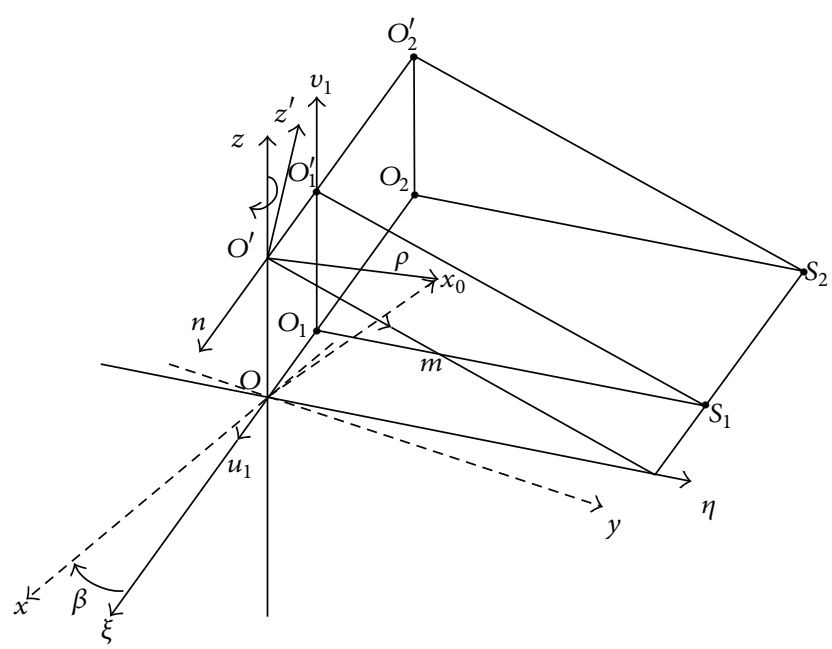

FIGURE 2: Definition of coordinate systems and geometrical parameters.

reconstructing formula deduction, reconstruction images are satisfying for a small cone angle, which are usually better for static objects. For dynamic process, there are some results in $[10,11]$.

\section{Nonconcyclic Dual-Source Circular Scan Mode with a Larger FOV}

In this section, we give a nonconcyclic dual-source circular cone beam scan mode with a larger FOV based on RT dualscan modes.

As shown in Figure 1, there are two pairs of X-ray sources and panel detectors. Let $S_{1}$ and $S_{2}$ denote the focuses of $\mathrm{X}$-ray sources. Let $D_{1}$ and $D_{2}$ denote panel detectors. In the nonconcyclic dual-source circular scan mode, two pairs of X-ray sources and panel detectors are fixed, and the inspected object is placed on the turntable. Now, we give this nonconcyclic dual-source scanning geometry. Firstly, the center of the turntable $O$ is located in the cone beam formed by $S_{1}$ and $D_{1}$. For some large inspected objects, one cone beam formed by $S_{1}$ and $D_{1}$ cannot completely cover its horizontal slice. So, secondly, we set $S_{2}$ and $D_{2}$ making $S_{1}, S_{2}$ and $D_{1}, D_{2}$ located at the same side of $O$, and two pairs of cone beams are parallel, as shown in Figure 1. Now, we give some relevant parameters to describe this scanning geometry. Let $R_{2}$ denote the distance from $S_{1}$ to $D_{1}$ (equal to the distance from $S_{2}$ to $D_{2}$ ). Let $R_{1}$ denote the distance from $S_{1}$ to the virtual panel detectors which is through $O$ and parallel to panel detectors $D_{1}$. Two pairs of panel detectors have the same size, where $L$ and $W$ denote the length and width of the panel detectors, respectively. To obtain sufficient projection data, the distance from $S_{2}$ to $S_{1}$ must be less than $L R_{1} /\left(2 R_{2}\right)$ [6]. According to the location of the three points $O, S_{1}$, and $S_{2}$, we know that distance from $S_{2}$ to $O$ is larger than the distance from $S_{1}$ to $O$. So two X-ray sources are nonconcyclic in this scanning geometry. The maximum horizontal FOV is $4 L\left(R_{2}-D\right) /\left(4 L+\sqrt{4 R_{2}^{2}+L^{2}}\right)$ in nonconcyclic scanning geometry [6], where $D$ is the distance from collimator of the detector to the crystal of the detector.

\section{Reconstruction Formula}

For the nonconcyclic dual-source circular scan mode above, we give the reconstruction formula in this section. We know that the key point to BPF algorithm is to obtain the Hilbert image from projections along some directions. The problem we face is how to weigh each set of the derivatives of two pairs of projection data to obtain two differentiated backprojection (DBP) images, and how to merge them into an entire DBP image that is related to the Hilbert image.

For the deduction of the reconstruction formula, we need give some denotations for the scan mode in Section 2. As shown in Figure 2, we establish 3D Cartesian coordinate system $O \xi \eta z$, where $z$-axis is the rotating axis. Like the deduction of many CT reconstruction formulas, we also adopt two virtual panel detectors whose centers are $O_{1}$ and $\mathrm{O}_{2}$, where the three points $\mathrm{O}_{1}, \mathrm{O}_{2}$, and $O$ are collinear. Let $O_{1}^{\prime}, O_{2}^{\prime}$ denote the projection point of $S_{1}, S_{2}$ to the upper side line of two virtual panel detectors, as shown in Figure 2. We need to establish rotating coordinate system Oxyz in the deduction, and let $\beta$ denote the rotating angle that is a clockwise angle from $\xi$-axis to $x$-axis. We set the $2 \mathrm{D}$ coordinate system $O_{i} u_{i} v_{i}(i=1,2)$ in two virtual panel detectors, where the direction vector of $u_{i}$ in $O x y z$ coordinate system is $\beta=(\cos \beta, \sin \beta, 0)$, and the direction vector of $v_{i}$ is the same direction with $z$-axis.

Let $f\left(\mathbf{x}_{0}\right)$ denote the density function of the inspected object where $\mathbf{x}_{0}=\left(x_{0}, y_{0}, z_{0}\right)$ is a reconstructed point on the inspected object. Let $b_{\theta}\left(\mathbf{x}_{0}\right)$ denote the DBP image of $f\left(\mathbf{x}_{0}\right)$, where $\theta$ is an angle measured from the $y$-axis anticlockwise in the plane $O x y$. Let $p_{i}\left(\beta, u_{i}, v_{i}\right)$ denote the projection data under X-ray source $S_{i}$, and $b_{\theta, i}\left(\mathbf{x}_{0}\right)$ denote the DBP image from $p_{i}\left(\beta, u_{i}, v_{i}\right)$. Let $h_{i}$ denote the distance between the points $O$ and $O_{i}$, and $h_{i}$ must satisfy $0<h_{1}<R_{1} L /\left(2 R_{2}\right)$ and $h_{2}<3 R_{1} L /\left(2 R_{2}\right)$ [6] that indicates that the projection 
area of the inspected objects under two X-ray sources is some what overlap. Let $\left(u_{0, i} v_{0, i}\right)$ denote the projection address of $\mathbf{x}_{0}$ in $O_{i} u_{i} v_{i}$ system under X-ray source $S_{i}$. Let $i=1,2$ above.

Now, using the idea of FDK algorithm, we deduct the formula of DBP image $b_{\theta}\left(\mathbf{x}_{0}\right)$ from the fan-beam DBP formula for RT multiscan modes $[5,6]$. The formula deduction is divided into two kinds of situations: (i) $\mathbf{x}_{0}=\left(x_{0}, y_{0}, z_{0}\right)$ is on the middle plane where $z_{0}=0$; (ii) $\mathbf{x}_{0}=\left(x_{0}, y_{0}, z_{0}\right)$ is on the off-middle plane where $z_{0} \neq 0$.

(i) If $\mathbf{x}_{0}$ is on the middle plane, we may directly obtain the cone beam DBP image $b_{\theta}\left(\mathbf{x}_{0}\right)$ from the fan-beam DBP [6] as follows:

$$
b_{\theta}\left(\mathbf{x}_{0}\right)=b_{\theta, 1}\left(\mathbf{x}_{0}\right)+b_{\theta, 2}\left(\mathbf{x}_{0}\right)
$$

where

$$
\begin{aligned}
& b_{\theta, 1}\left(\mathbf{x}_{0}\right) \\
& =\int_{0}^{2 \pi} \frac{R_{1}}{\left(R_{1}-\mathbf{x}_{0} \cdot \boldsymbol{\beta}^{\perp}\right)^{2}} \frac{\mathrm{d}}{\mathrm{d} u_{1}} \\
& \times\left\{\left[k_{\varepsilon}\left(\frac{R_{1}\left(u_{1}+h_{1}\right)}{\sqrt{R_{1}^{2}+u_{1}^{2}}}\right)\right.\right. \\
& \left.-k_{\varepsilon}\left(\frac{R_{1}\left(u_{1}+h_{1}\right)}{\sqrt{R_{1}^{2}+u_{1}^{2}}}-T_{1}\right)\right] \\
& \times \frac{R_{1}^{2}-h_{1} u_{1}}{\sqrt{R_{1}^{2}+u_{1}^{2}}} \times p_{1}\left(\beta, u_{1}, 0\right) \\
& \times \operatorname{sgn}\left(\operatorname { s i n } \left(\beta+\tan ^{-1}\right.\right. \\
& \left.\left.\left.\times \frac{u_{1}}{R_{1}}-\theta\right)\right)\right\}\left.\right|_{u_{1}=u_{0,1}} \mathrm{~d} \beta, \\
& b_{\theta, 2}\left(\mathbf{x}_{0}\right)=\int_{0}^{2 \pi} \frac{R_{1}}{\left(R_{1}-\mathbf{x}_{0} \cdot \boldsymbol{\beta}^{\perp}\right)^{2}} \frac{\mathrm{d}}{\mathrm{d} u_{2}} \\
& \times\left\{\left(k_{\varepsilon}\left(\frac{R_{1}\left(u_{2}+h_{2}\right)}{\sqrt{R_{1}^{2}+u_{2}^{2}}}-T_{1}\right)\right)\right. \\
& \times \frac{R_{1}^{2}-h_{2} u_{2}}{\sqrt{R_{1}^{2}+u_{2}^{2}}} \times p_{2}\left(\beta, u_{2}, 0\right) \\
& \left.\times \operatorname{sgn}\left(\sin \left(\beta+\tan ^{-1} \frac{u_{2}}{R_{1}}-\theta\right)\right)\right\}\left.\right|_{u_{2}=u_{0,2}} \mathrm{~d} \beta
\end{aligned}
$$

where

$$
\begin{gathered}
\boldsymbol{\beta}^{\perp}=(-\sin \beta, \cos \beta, 0), \\
u_{0, i}=\frac{R_{1}\left(\mathbf{x}_{0} \cdot \boldsymbol{\beta}-h_{i}\right)}{R_{1}-\mathbf{x}_{0} \cdot \boldsymbol{\beta}^{\perp}}, \quad i=1,2,
\end{gathered}
$$

$$
\begin{gathered}
T_{1}=\frac{R_{1}\left(h_{2}+h_{1}\right)}{\sqrt{4 R_{1}^{2}+\left(h_{2}-h_{1}\right)^{2}}}, \\
k_{\varepsilon}(r)=\frac{1}{2 \varepsilon} \int_{-\infty}^{\infty}\left(\operatorname{sgn}\left(r^{\prime}\right)+1\right) w\left(\frac{r-r^{\prime}}{\varepsilon}\right) \mathrm{d} r^{\prime},
\end{gathered}
$$

where $\varepsilon$ is a small positive number that is determined by the projection overlapping area, and $w(r)$ is a mollification kernel function as follows:

$$
\begin{gathered}
w(r)= \begin{cases}C \operatorname{Exp}\left(\frac{-1}{1-r^{2}}\right), & |r|<1, \\
0, & |r| \geq 1,\end{cases} \\
C=\left(\int_{-1}^{1} \operatorname{Exp}\left(\frac{-1}{1-r^{2}}\right) \mathrm{d} r\right)^{-1} .
\end{gathered}
$$

(ii) If $\mathbf{x}_{0}$ is on an off-middle plane, for obtaining $b_{\theta}\left(\mathbf{x}_{0}\right)$ we need to define the oblique surface which is through the points $S_{1}, S_{2}$, and $\mathbf{x}_{0}$. Let $O^{\prime}$ denote the intersection point between $z$-axis and the oblique surface, and we know $O^{\prime}=\left(0,0, v_{1}\right)$ from Figure 2 above. Now, we establish the coordinate system and give the relevant parameters in the oblique surface. Let $n \mathrm{O}^{\prime} m$ denote the coordinate system in the oblique surface, where $n, m$ is the same direction with the vectors $\mathbf{u}_{1}$ and $\mathbf{O}_{1}^{\prime} \mathbf{S}_{1}$, respectively. Let $\rho, \beta^{\prime}$ denote a vector from $O^{\prime}$ to $\mathbf{x}_{0}$ and an angle variable in the oblique surface, respectively, and $R_{1}^{\prime}$ denote the distance from $S_{1}$ to $O^{\prime}$ in the oblique surface.

We know that X-ray source is regarded as a fulcrum in FDK algorithm, and an off-middle plane is approximately obtained by inclining the middle plane. Now, based on the idea of FDK algorithm, we give the steps of deduction of $b_{\theta}\left(\mathbf{x}_{0}\right)$ in the off-middle plane as follows: (i) writing $\delta b_{\theta}\left(\mathbf{x}_{0}\right)=$ $\delta b_{\theta, 1}\left(\mathbf{x}_{0}\right)+\delta b_{\theta, 2}\left(\mathbf{x}_{0}\right)$ using the variables in the oblique surface; (ii) finding the relation between $\delta \beta$ and $\delta \beta^{\prime}$, where $\delta \beta$ is the rotation angle increment in the middle plane, and $\delta \beta^{\prime}$ is the rotation angle increment in the oblique plane; (iii) finding the relation between $\mathbf{x}_{0}$ and $\rho$; (iv) calculating $R_{1}^{\prime}$; (v) obtaining $b_{\theta}\left(\mathbf{x}_{0}\right)$ by accumulating all $\delta b_{\theta}\left(\mathbf{x}_{0}\right)$ for the angle variable $\beta$.

Without loss of generality, we give the deduction of $b_{\theta, 1}\left(\mathbf{x}_{0}\right)$ referring to the steps above. From the formula (2), making use of the parameters in the oblique surface $n \mathrm{O}^{\prime} \mathrm{m}$, we obtain.

$$
\begin{aligned}
\delta b_{\theta, 1}\left(\mathbf{x}_{0}\right)= & \delta \beta^{\prime} \frac{\sqrt{R_{1}^{\prime 2}-h_{1}^{2}}}{\left(\sqrt{R_{1}^{\prime 2}-h_{1}^{2}}-\rho \cdot m\right)^{2}} \frac{\mathrm{d}}{\mathrm{d} u_{1}} \\
& \times\left\{\left[k_{\varepsilon}\left(\frac{R_{1}\left(u_{1}+h_{1}\right)}{\sqrt{R_{1}^{2}+u_{1}^{2}}}\right)\right.\right.
\end{aligned}
$$




$$
\begin{aligned}
& \left.-k_{\varepsilon}\left(\frac{R_{1}\left(u_{1}+h_{1}\right)}{\sqrt{R_{1}^{2}+u_{1}^{2}}}-T_{1}\right)\right] \\
& \times \frac{\left(R_{1}^{\prime 2}-h_{1}^{2}\right)-h_{1} u_{1}}{\sqrt{R_{1}^{\prime 2}-h_{1}^{2}+u_{1}^{2}}} \times p_{1}\left(\beta, u_{1}, v_{1}\right) \\
& \left.\times \operatorname{sgn}\left(\sin \left(\beta+\tan ^{-1} \frac{u_{1}}{R_{1}}-\theta\right)\right)\right\}\left.\right|_{u_{1}=u_{0,1}, v_{1}=v_{0,1}},
\end{aligned}
$$

(6)

where $v_{0,1}=\left(R_{1} \times z_{0}\right) /\left(R_{1}-\mathbf{x}_{0} \times \boldsymbol{\beta}^{\perp}\right)$. Obviously, $\rho$ is $\mathbf{x}_{0}$, when $z_{0}=0$.

We can obtain the relation between $\delta \beta$ and $\delta \beta^{\prime}$ from Figure 2 as

$$
\delta \beta^{\prime}=\delta \beta \frac{\sqrt{R_{1}^{2}+h_{1}^{2}}}{\sqrt{R_{1}^{2}+h_{1}^{2}+v_{1}^{2}}},
$$

and the relation between $\mathbf{x}_{0}$ and $\rho$ as

$$
\mathbf{x}_{0}=\rho+v_{1} z
$$

Since $\rho$ is in the plane $n O^{\prime} m$, we can obtain

$$
\begin{gathered}
\rho \cdot(n \times m)=0, \\
\rho \cdot m=\frac{R_{1}^{\prime}}{\sqrt{R_{1}^{2}+h_{1}^{2}}} \mathbf{x}_{0} \cdot \boldsymbol{\beta}^{\perp} .
\end{gathered}
$$

We easily calculate the distance $R_{1}^{\prime}$ from $S_{1}$ to the detectors in the oblique surface,

$$
R_{1}^{\prime}=\left|\mathbf{S}_{1} \mathbf{O}^{\prime}\right|=\sqrt{R_{1}^{2}+h_{1}^{2}+v_{1}^{2}}
$$

Now, we substitute formulas (7), (8), (9), and (10) to (6) and obtain $b_{\theta, 1}\left(\mathbf{x}_{0}\right)$ by accumulating all $\delta b_{\theta}\left(\mathbf{x}_{0}\right)$ for the angle variable $\beta$ as

$$
\begin{aligned}
b_{\theta, 1}\left(\mathbf{x}_{0}\right)= & \int_{0}^{2 \pi} U_{1} \frac{\mathrm{d}}{\mathrm{d} u_{1}} \\
& \times\left\{\left[k_{\varepsilon}\left(\frac{R_{1}\left(u_{1}+h_{1}\right)}{\sqrt{R_{1}^{2}+u_{1}^{2}}}\right)\right.\right.
\end{aligned}
$$

$$
\begin{aligned}
& \left.-k_{\varepsilon}\left(\frac{R_{1}\left(u_{1}+h_{1}\right)}{\sqrt{R_{1}^{2}+u_{1}^{2}}}-T_{1}\right)\right] \\
& \times \frac{\left(R_{1}^{2}+v_{1}^{2}\right)-h_{1} u_{1}}{\sqrt{R_{1}^{2}+v_{1}^{2}+u_{1}^{2}}} \times p_{1}\left(\beta, u_{1}, v_{1}\right) \\
& \left.\times \operatorname{sgn}\left(\sin \left(\beta+\tan ^{-1} \frac{u_{1}}{R_{1}}-\theta\right)\right)\right\}\left.\right|_{u_{1}=u_{0,1}, v_{1}=v_{0,1}} \mathrm{~d} \beta,
\end{aligned}
$$

where

$$
\begin{gathered}
U_{1}=\frac{\left(R_{1}^{2}+h_{1}^{2}\right)^{3 / 2} \sqrt{R_{1}^{2}+v_{1}^{2}}}{M_{1} \sqrt{R_{1}^{2}+h_{1}^{2}+v_{1}^{2}}} \\
M_{1}=\left(\sqrt{\left(R_{1}^{2}+v_{1}^{2}\right)\left(R_{1}^{2}+h_{1}^{2}\right)}-\sqrt{R_{1}^{2}+h_{1}^{2}+v_{1}^{2}} \mathbf{x}_{0} \cdot \boldsymbol{\beta}^{\perp}\right)^{2} .
\end{gathered}
$$

Similarly, we can obtain

$$
\begin{aligned}
b_{\theta, 2}\left(\mathbf{x}_{0}\right)=\int_{0}^{2 \pi} & U_{2} \frac{\mathrm{d}}{\mathrm{d} u_{2}} \\
\times & \left\{\left(k_{\varepsilon}\left(\frac{R_{1}\left(u_{2}+h_{2}\right)}{\sqrt{R_{1}^{2}+u_{2}^{2}}}-T_{1}\right)\right)\right. \\
& \times \frac{\left(R_{1}^{2}+v_{2}^{2}\right)-h_{2} u_{2}}{\sqrt{R_{1}^{2}+v_{2}^{2}+u_{2}^{2}} \times p_{2}\left(\beta, u_{2}, v_{2}\right)} \\
& \left.\times \operatorname{sgn}\left(\sin \left(\beta+\tan ^{-1} \frac{u_{2}}{R_{1}}-\theta\right)\right)\right\}\left.\right|_{u_{2}=u_{0,2}, v_{2}=v_{0,2}} \mathrm{~d} \beta,
\end{aligned}
$$

where

$$
\begin{gathered}
U_{2}=\frac{\left(R_{1}^{2}+h_{2}^{2}\right)^{3 / 2} \sqrt{R_{1}^{2}+v_{2}^{2}}}{M_{2} \sqrt{R_{1}^{2}+h_{2}^{2}+v_{2}^{2}}}, \\
M_{2}=\left(\sqrt{\left(R_{1}^{2}+v_{2}^{2}\right)\left(R_{1}^{2}+h_{2}^{2}\right)}\right. \\
\left.-\sqrt{R_{1}^{2}+h_{2}^{2}+v_{2}^{2}} \mathbf{x}_{0} \cdot \boldsymbol{\beta}^{\perp}\right)^{2},
\end{gathered}
$$

where $v_{0, i}=\left(R_{1} \times z_{0}\right) /\left(R_{1}-\mathbf{x}_{0} \times \beta^{\perp}\right)$. When $z_{0}=0$, the formulas (11) and (13) are (2) and (3), respectively.

We need two steps for reconstructing $f(\mathbf{x})$. Firstly, we need to obtain the Hilbert image $H_{\theta} f(\mathbf{x})$ of each layer according to the reference [8]. Using the relation of the 


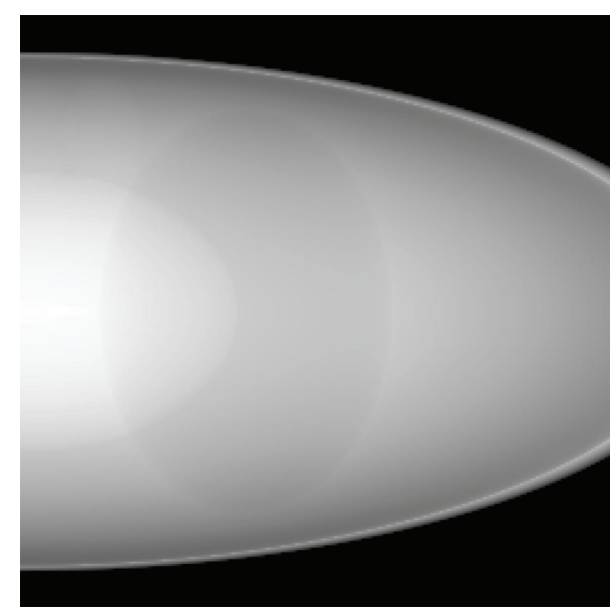

(a)

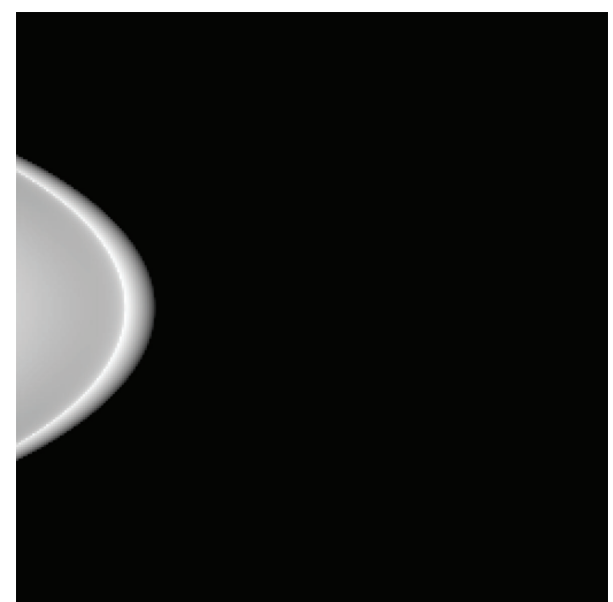

(b)

FIgure 3: Two DR images under the 100th project angle, (a) DR image from the cone beam formed by the X-ray source $S_{1}$ and the panel detectors $D_{1}$; (b) DR image from the cone beam formed by the X-ray $S_{2}$ and the panel detectors $D_{2}$.

Hilbert image and the DBP image, we can obtain from the formulas (1), (2), (3), (11), and (13),

$$
\left.H_{\theta} f(\mathbf{x})\right|_{z=z_{0}}=-\frac{\left.b_{\theta}(\mathbf{x})\right|_{z=z_{0}}}{2 \pi},
$$

where $\mathbf{x}=(x, y, z)$ is an arbitrary point on the inspected object. Secondly, making use of the virtual trajectories and virtual PI-lines in $[12,13]$, we can obtain the following formula based on the finite inversion formula of Hilbert transform:

$$
\begin{aligned}
\left.f(\mathbf{x})\right|_{z=z_{0}} & \\
= & \frac{-1}{\sqrt{\left(\mathbf{x} \cdot \boldsymbol{\theta}^{\prime \perp}-L_{t, z}\right)\left(U_{t, z}-\mathbf{x} \cdot \boldsymbol{\theta}^{\prime \perp}\right)}} \\
& \times\left(\int_{L_{t, z}}^{U_{t, z}} \sqrt{\left(s-L_{t, z}\right)\left(U_{t, z}-s\right)}\right. \\
& \times \frac{H_{\theta} f\left(\left(\mathbf{x} \cdot \boldsymbol{\theta}^{\prime}\right) \boldsymbol{\theta}^{\prime}+s \boldsymbol{\theta}^{\prime \perp}+z(0,0,1)\right)}{\pi\left(\mathbf{x} \cdot \boldsymbol{\theta}^{\prime \perp}-s\right)} \mathrm{d} s \\
& \left.+C_{t, z}\right)\left.\right|_{z=z_{0}},
\end{aligned}
$$

where $\boldsymbol{\theta}^{\prime}=(\cos \theta, \sin \theta, 0), \boldsymbol{\theta}^{\prime \perp}=(-\sin \theta, \cos \theta, 0)$, and the constants $L_{t, z}, U_{t, z}$, and $C_{t, z}$ relate to $\mathbf{x} \cdot \boldsymbol{\theta}^{\prime}=t$ and $z$. We can obtain $C_{t, z}$ from the integral of $f(\mathbf{x})$ along the virtual PI-lines [14]. However, since the PI-lines are virtual except the middle plane, we cannot obtain an accurate $C_{t, z}$. So the proposed reconstruction formula is approximated on the offmiddle planes.

\section{Numerical Experiments}

To validate our algorithm, we perform some numerical experiments with simulated data in this section. 3D Shepp-Logan
TABLE 1: Parameters of 3D Shepp-Logan phantom.

\begin{tabular}{ccccccccc}
\hline$N$ & $x_{c}$ & $y_{c}$ & $z_{c}$ & $a$ & $b$ & $c$ & $\begin{array}{c}\alpha \\
(\text { degree })\end{array}$ & $\begin{array}{c}\text { Density } \\
\text { value }\end{array}$ \\
\hline 1 & 0 & 0 & 0 & 97.9 & 73.4 & 26.8 & 0 & 1000.0 \\
2 & 0 & 0 & 0 & 93.0 & 70.5 & 26.2 & 0 & 300.0 \\
3 & -23.4 & 0 & 0 & 17.0 & 43.6 & 20.8 & 108 & -200.0 \\
4 & 23.4 & 0 & 0 & 11.7 & 33.0 & 20.8 & 72 & -200.0 \\
5 & 0 & 37.2 & 0 & 26.6 & 22.4 & 14.9 & 0 & 200.0 \\
6 & 0 & 10.6 & 0 & 4.9 & 4.9 & 1.4 & 0 & 200.0 \\
7 & -6.4 & -69.2 & 0 & 2.5 & 4.9 & 0.6 & 0 & 200.0 \\
8 & 6.4 & -69.2 & 0 & 2.5 & 4.9 & 0.6 & 90 & 200.0 \\
9 & 8.5 & 0 & 18.6 & 5.9 & 4.3 & 6.0 & 0 & 200.0 \\
10 & 0 & 0 & 18.6 & 5.9 & 5.9 & 6.0 & 0 & 200.0 \\
\hline
\end{tabular}

phantom is used to get the simulated data. The parameters of the phantom in 3D Cartesian coordinate system $O x y z$ are listed in Table 1 , where $\left(x_{c}, y_{c}, z_{c}\right)$ is the center coordinate of an ellipsoid, and three variables $a, b$ and $c$ denote the length of three half axis of an ellipsoid, and $\alpha$ is the rotating angle of an ellipsoid in the plane $x \mathrm{O} y$. The parameters of two pairs of $\mathrm{X}$-ray sources and panel detectors in the scanning geometry are the same as follows: $R_{1}=1426.0 \mathrm{~mm}, R_{2}=1800.0 \mathrm{~mm}$, and the length and width of the panel detectors $L=W=$ $77.1 \mathrm{~mm}$, which is composed of $257 \times 257$ detector cells.

According to the parameters above, we can calculate the horizontal diameter of the maximum FOV formed by a set of X-ray source and panel detector is $60.82 \mathrm{~mm}$. From the parameters of 3D Shepp-Logan phantom in Table 1, we calculate the longest axis of ellipsoid along $x$-axis to be $97.9 \mathrm{~mm}$ which is greater than $60.82 \mathrm{~mm}$. So we cannot reconstruct its CT image using Cardiac dual-source CT scanning geometry. For acquiring the complete CT data of 


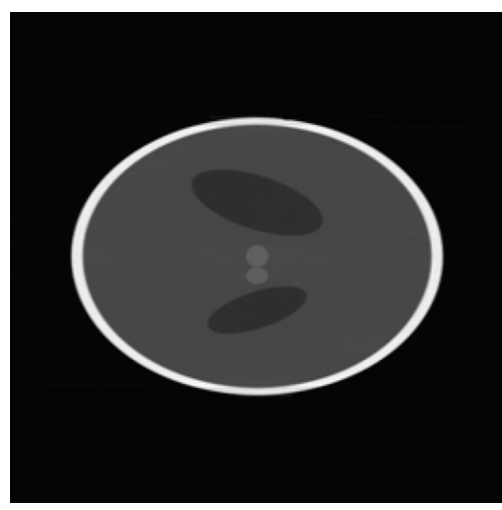

(a)

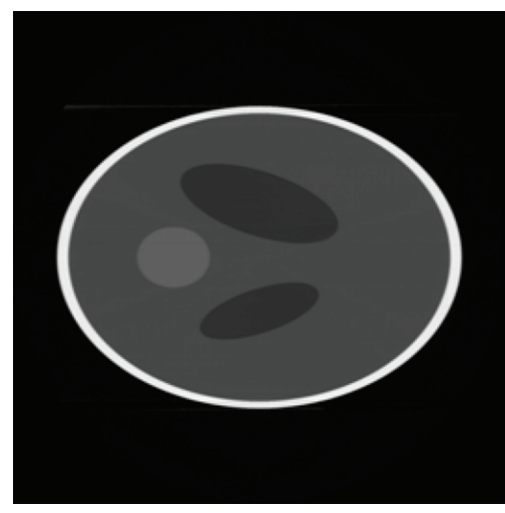

(b)

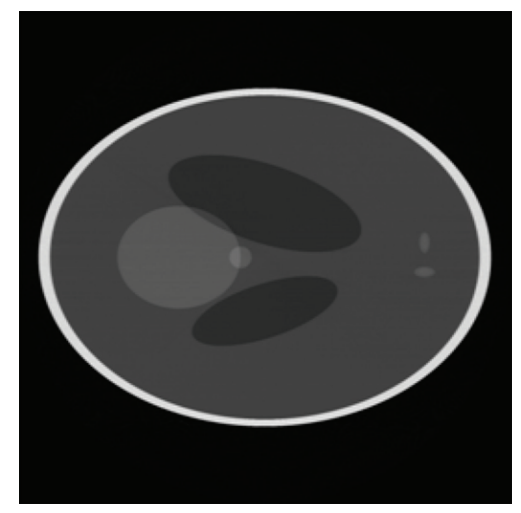

(c)

FIgURE 4: Three reconstruction images of the horizontal slices, (a) $z=-16.8 \mathrm{~mm}$, (b) $z=0$, and (c) $z=12.8 \mathrm{~mm}$.

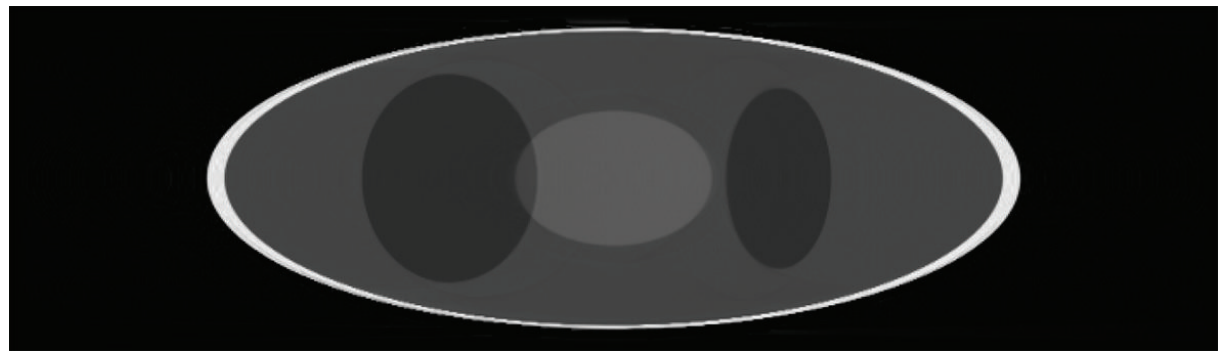

(a)

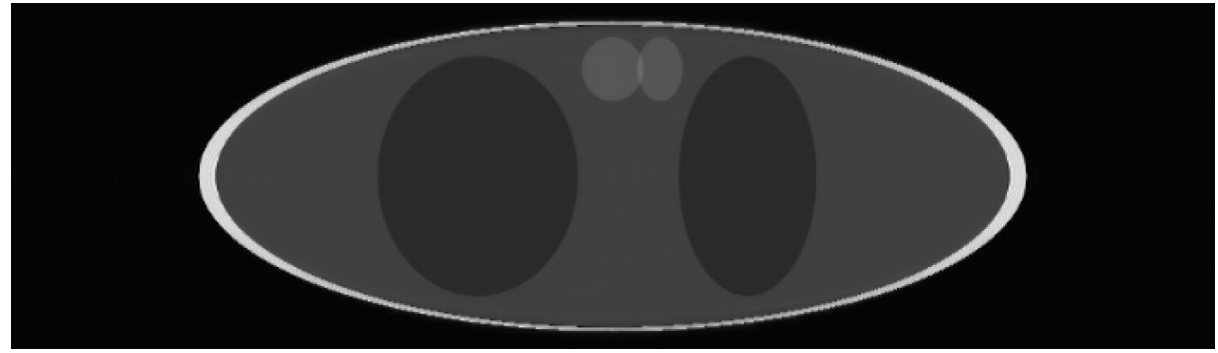

(b)

Figure 5: Two reconstruction images of the slices perpendicular to $x$-axis, (a) $x=-20.4 \mathrm{~mm}$, (b) $x=2.1 \mathrm{~mm}$.

the 3D Shepp-Logan phantom, we adapt the nonconcyclic dual-source circular cone beam scanning geometry with a larger FOV in Section 2. In this scanning geometry, we set $h_{1}=30.42 \mathrm{~mm}$ and $h_{2}=91.26 \mathrm{~mm}$ which satisfie the conditions above. Each panel detector takes 720 projections within the angle range from 0 to $2 \pi$. Two digital radiography (DR) images under the 100th projection angle are shown in Figure 3. In CT image reconstruction, we use $\theta=0$ as the direction of Hilbert transform, and $\varepsilon=0.24$. We reconstruct the CT images from the formulas (15) and (16). Three-image matrix of a horizontal slice is $701 \times 701$, as shown in Figure 4 . Two-image matrix of a slice perpendicular to $x$-axis is $701 \times 200$, as shown in Figure 5 . Two-image matrix of a slice perpendicular to $y$-axis is $701 \times 200$, as shown in Figure 6. For two three-dimensional projection data whose size is $720 \times 257 \times 257$, CT image reconstruction takes about
1200 seconds by using CPU and 17.778 seconds by using GPU, where the size of CT image is $701 \times 701 \times 200$.

\section{Conclusion}

In this work we give nonconcyclic dual-source circular cone beam scan mode with a larger FOV, and deduce the reconstruction formula. Using two pairs of projections obtained from this nonconcyclic dual-source scanning geometry, the FOV is enlarged effectively in the same equipment condition without data rebinning. It is because of the fact that for the real CT system, the flux output from X-ray source is not isotropic, and then the data acquired in this scanning mode using small panel detectors are relative to more uniform intensity of the flux output than a large one. The experiment 


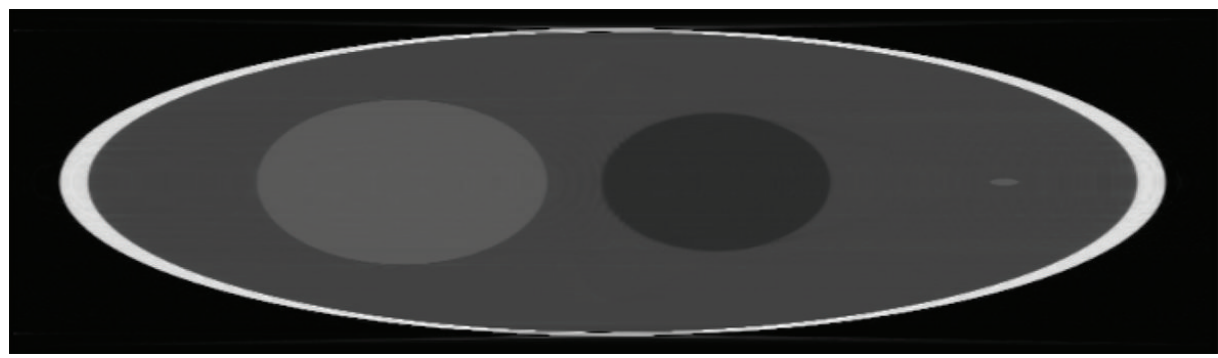

(a)

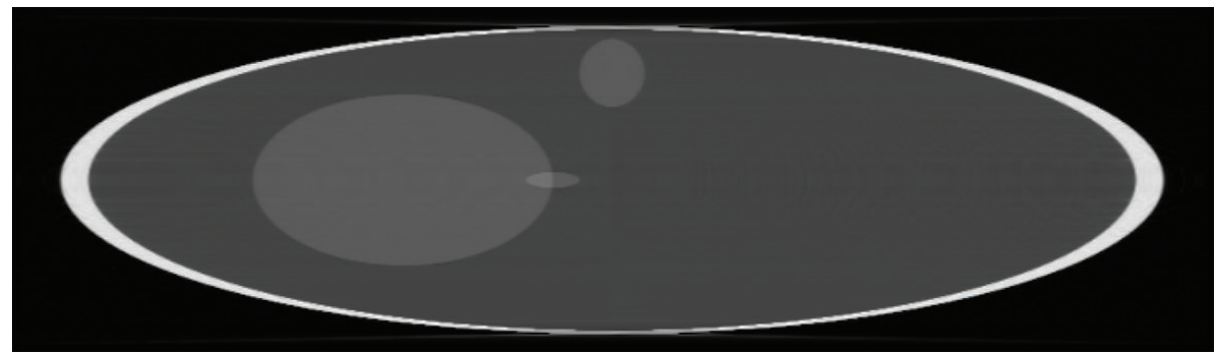

(b)

FigurE 6: Two reconstruction images of the slices perpendicular to $y$-axis, (a) $y=-6.0 \mathrm{~mm}$, (b) $y=0.9 \mathrm{~mm}$.

confirmed that our reconstruction method is effective, when a cone angle is small.

\section{Acknowledgments}

This work was supported in part by three grants from the National Natural Science Foundation of China (61201430, 61002041, and 61201431), International Scientific and Technological Cooperation Program of Shenzhen (Grant JC201105190923A), China Postdoctoral Science Foundation and Shandong province Postdoctoral Innovation Foundation.

\section{References}

[1] E. A. Sivers, "Use of multiple CT scans to accommodate large objects and stretch dynamic range of detectability," Nuclear Instruments and Methods in Physics B, vol. 99, pp. 761-764, 1995.

[2] E. A. Sivers, W. A. Snyder, S. A. Ellingson et al., "CT Multiscan: using small area detectors to image large components," Journal of Engineering for Gas Turbines and Power, vol. 118, pp. 711-716, 1996.

[3] Y. Q. Sun, Researches on several application problems in industrial CT [M.S. thesis], Capital Normal University, Beijing, China, 2006.

[4] F. Zhao, H. N. Lu, and C. L. Sun, "New scan mode for 2D-CT and its reconstruction algorithm," Optech, vol. 32, pp. 284-289, 2006.

[5] M. Chen, H. T. Zhang, and P. Zhang, "BPF-based reconstruction algorithm for multiple rotation-translation scan mode," Progress in Natural Science, vol. 18, pp. 209-216, 2008.

[6] M. Chen, H. T. Zhang, D. F. Chen, and P. Zhang, "Reconstruction algorithm for unilateral off-centered rotation translation multi-scans," NDT, China, vol. 31, pp. 29-34, 2009.
[7] Y. Zou and X. Pan, "Exact image reconstruction on PI-lines from minimum data in helical cone-beam CT," Physics in Medicine and Biology, vol. 49, pp. 941-959, 2004.

[8] F. Noo, R. Clackdoyle, and J. D. Pack, "A two-step Hilbert transform method for 2D image reconstruction," Physics in Medicine and Biology, vol. 49, pp. 3903-3923, 2004.

[9] L. A. Feldkamp, L. C. Davis, and J. W. Kress, "Practical conebeam algorithm," Journal of the Optical Society of America A, vol. 1, pp. 612-619, 1984.

[10] Y. W. Zhang, T. Y. Chai, Z. M. Li, and C. Y. Yang, "Modeling and monitoring of dynamic processes," IEEE Transactions on Neural Networks and Learning System, vol. 23, pp. 277-284, 2012.

[11] Y. W. Zhang, H. Zhou, S. J. Qin, and T. Y. Chai, “Decentralized fault diagnosis of large-scale processes using multiblock kernel partial least squares," IEEE Transactions on Industrial Informatics, vol. 6, no. 1, pp. 3-10, 2010.

[12] Y. Zou, X. Pan, and E. Y. Sidky, "Image reconstruction in regions-of-interest from truncated projections in a reduced fanbeam scan," Physics in Medicine and Biology, vol. 50, pp. 13-27, 2005.

[13] L. Yu, Y. Zou, E. Y. Sidky, C. A. Pelizzari, P. Munro, and X. Pan, "Region of interest reconstruction from truncated data in circular cone-beam CT," IEEE Transactions on Medical Imaging, vol. 25, no. 7, pp. 869-881, 2006.

[14] Y. Sidky Emil, Y. Zou, and X. Pan, "Minimum data image reconstruction algorithms with shift-invariant filtering for helical, cone-beam CT," Physics in Medicine and Biology, vol. 50, pp. 1643-1657, 2005. 


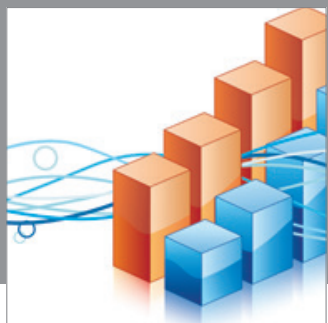

Advances in

Operations Research

mansans

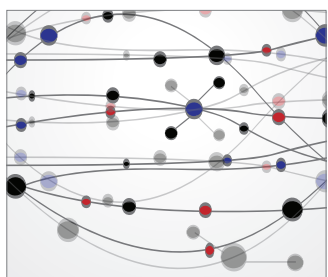

The Scientific World Journal
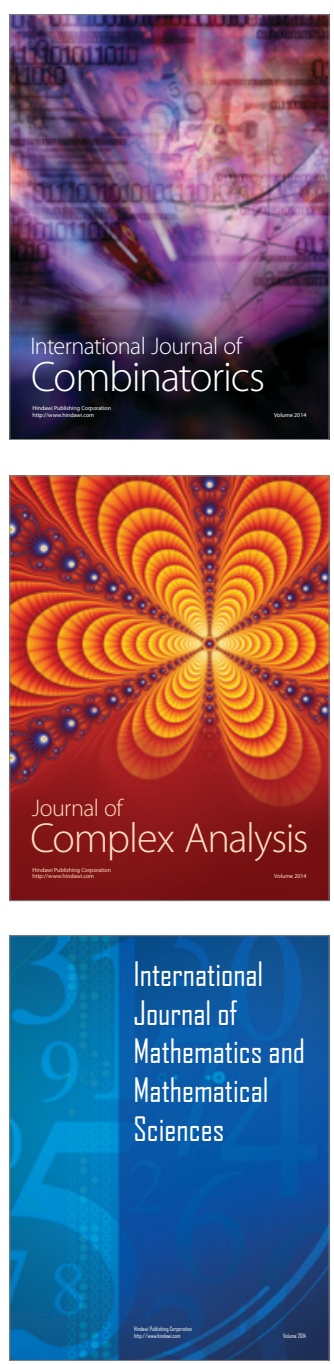
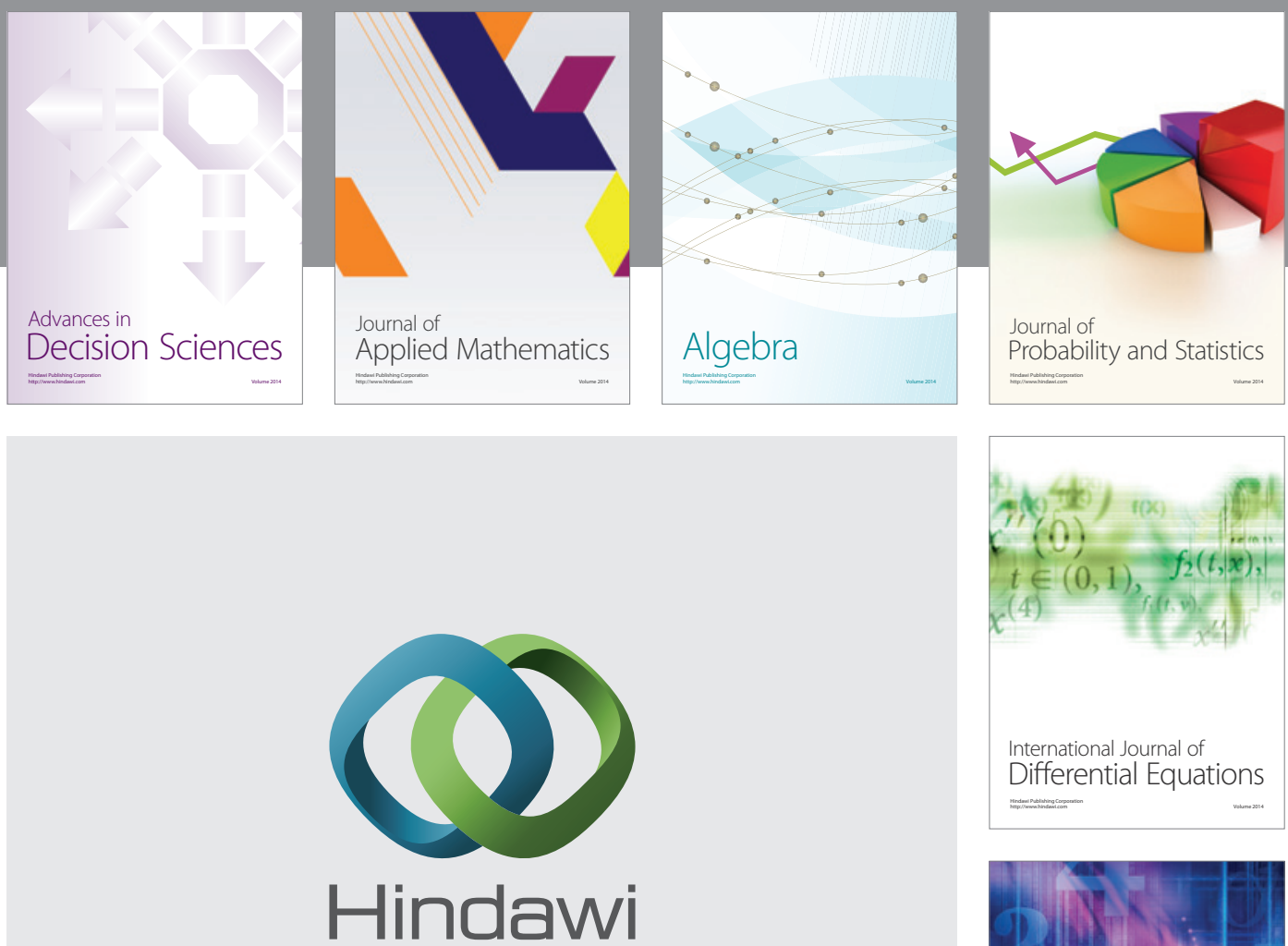

Submit your manuscripts at http://www.hindawi.com
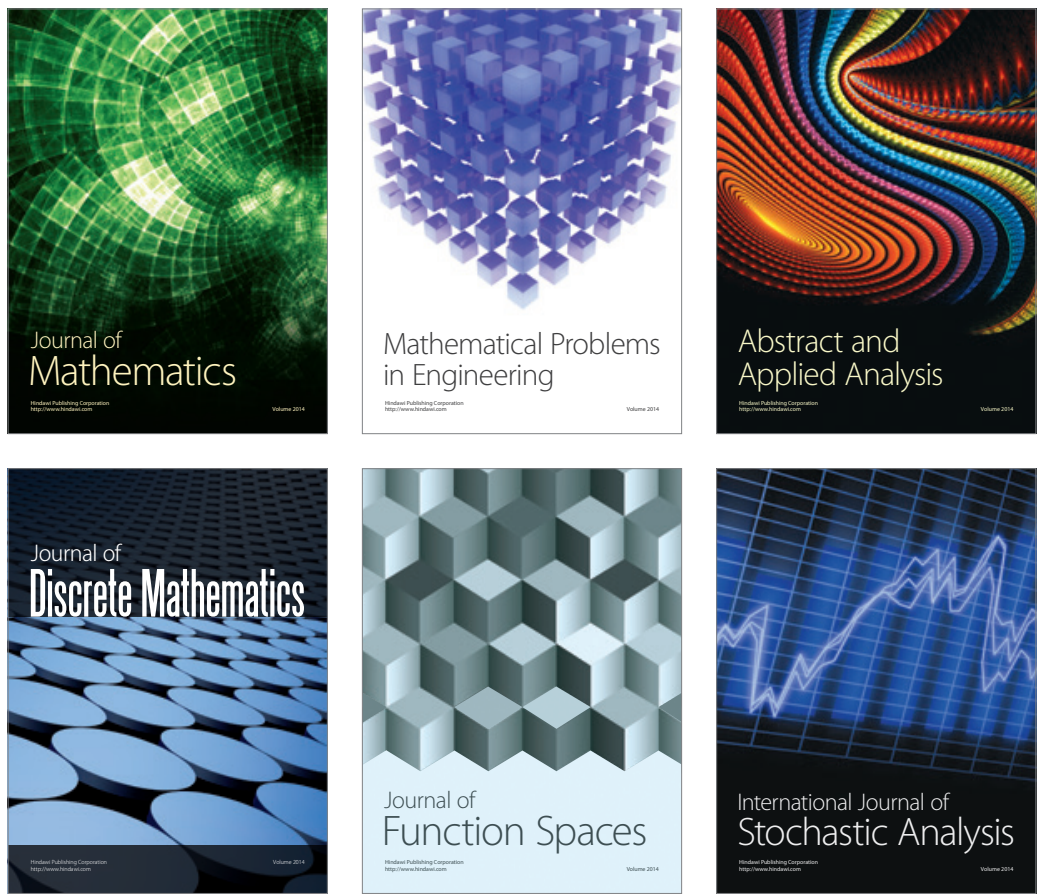

Journal of

Function Spaces

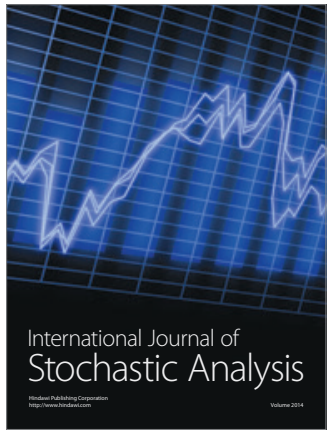

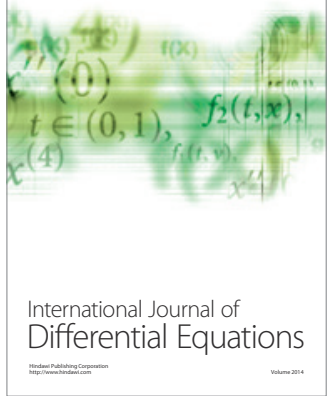
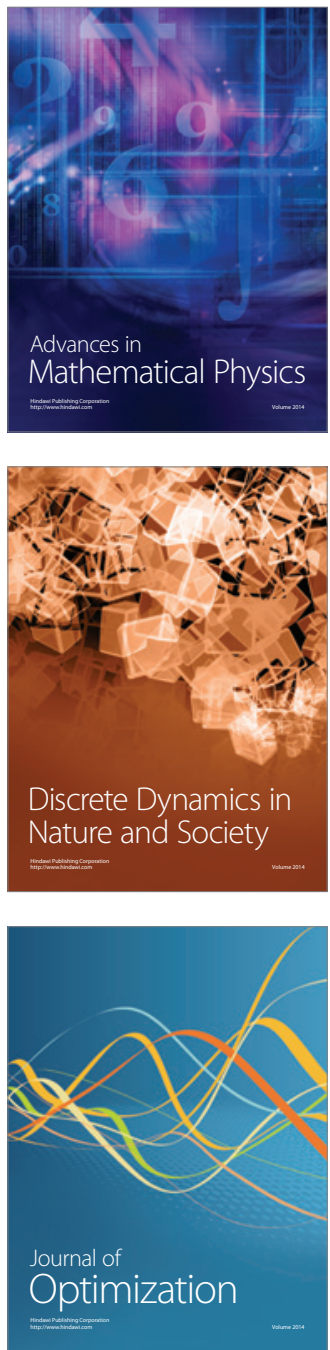